\title{
Free Vibrational Response of Single-Layered Graphene Sheets Embedded in an Elastic Matrix using Different Nonlocal Plate Models
}

\author{
Babak SAFAEI, A.M. FATTAHI* \\ *Department of Mechanical Engineering, Tabriz Branch, Islamic Azad University, Tabriz, Iran, \\ E-mail: a.fattahi@iaut.ac.ir

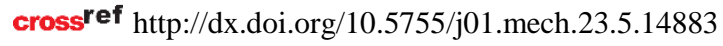

\section{Introduction}

Owing to their outstanding mechanical, electrical, and chemical properties, the family of carbon allotropes including carbon nanotubes, graphene sheets and fullerenes are becoming increasingly important in the emerging field of nanoscience and nanotechnology [1-2]. Technical difficulties in conducting experiments at the nanoscale make it necessary to have recourse to theoretical approaches for investigating the behaviour of nanostructures.

Based on the classical plate theory (CLPT), Kitipornchai et al. [3] investigated the vibration response of multi-layered graphene sheets (MLGSs) with simply-supported boundary conditions using a continuum model. They proposed an explicit formula for the van der Waals interaction between any two sheets in a MLSG. Liew and his coworkers [4] proposed a continuum model to analyse the vibrations of MLSG embedded in an elastic matrix.

Because of not having the capability of considering the size-effects, the implementation the classical continuum models to predict the behaviour of nanostructures becomes controversial. Hence, the extension of the continuum mechanics to accommodate the size dependence of nanostructures is a topic of major concern. Modified continuum models are one of the most applied theoretical approaches for the investigation of nanomechanics due to their computational efficiency and the capability to produce accurate results which are comparable to those of atomistic models. The application of nonlocal continuum mechanics allowing for the small scale effects to the vibrational analysis of nanomaterials has been recommended by many research workers.

Azizi et al. [5-6] using carbon nanotubes as reinforcing fibres have been performed to address the exceptional mechanical and electrical properties of nanotubebased composites. Vibration analysis of MLGSs embedded in polymer matrix was investigated by Pradhan and Phadikar [7] using nonlocal continuum mechanics.

Continuing with the vibration problems, Pradhan et al. [8] developed a single-elastic beam model to analyse the thermal vibration of CNTs based on thermal elasticity mechanics, and nonlocal elasticity theory. The effect of nonlocal scale parameter on the wave propagation in multiwalled carbon nanotubes was represented by Narendar and Gopalakrishnan [9]. Murmu and Pradhan [10] studied the vibration characteristics of single-walled carbon nanotubes (SWCNTs) based upon a nonlocal shell model.

There are so many other researches in which the behaviours of nanostructures under various loading conditions have been predicted based on nonlocal elasticity continuum models [11-20] which indicate the wide application of this type of modified continuum mechanics in nanomechanics.

In many cases such as polymer nanocomposites, the nanostructures can be embedded in an elastic surrounding medium. This elastic medium is generally simulated using Winkler foundation model [21]. Based on this type of foundation, the elastic matrix is represented as a series of closely spaced, vertical linear elastic springs. However, this model does not have the capability to consider the continuity of the medium. A more practical modelling of elastic foundation can be implemented using Pasternak foundation model [22] which regards both normal pressure and transverse shear stress using two modulus parameters corresponding to each one. The Pasternak foundation model was used by Pradhan and Murmu [23], and Liew et al. [24] to simulate the interaction of the elastic medium with graphene sheet and successful results were obtained to show the physically realistic application of this type of foundation modelling.

According to the above literature review, it can be seen that the investigation of size-effects on behaviours of nanostructures has assigned so many researches. In the current study, the free vibrational response of SLGSs embedded in an elastic medium is investigated based on various nonlocal plate models. Both Winkler and Pasternak elastic foundation models are employed to represent the surrounding elastic matrix. Closed-form analytical solution is developed to obtain explicit formulas to obtain the natural frequencies of SLGSs corresponding to each type of nonlocal plate theory through exact solution for the governing differential equations. Selected numerical results are presented to show the influence of nonlocality, elastic foundation, type of nonlocal plate theory and side length of square SLGSs in detail.

\section{Overview of various plate theories}

\subsection{Introduction}

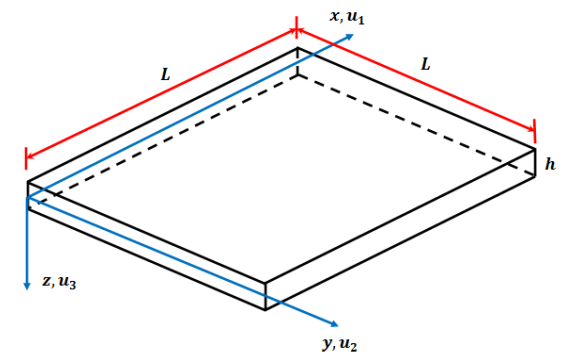

Fig. 1 Schematic of a nanoplate: kinematic parameters, coordinate system and geometry 
To represent the behaviour of plates, there are different plate theories. As it can be seen from Fig. 1, consider a uniform square nanoplate with the side length $L$ and thickness $h$. A coordinate system $(x, y, z)$ is introduced at the one corner of the midplane of the nanoplate, whereas the $x$ axis is taken along the length of the nanoplate, the $y$ axis in the width direction and the $\mathrm{z}$ axis is taken along the depth (thickness) direction. The displacement components $\left(u_{1}, u_{2}, u_{3}\right)$ along the axes $(x, y, z)$ can be written in a general form as:

$$
\begin{aligned}
& u_{1}=-z \frac{\partial w}{\partial x}+\psi(z)\left(\frac{\partial w}{\partial x}+\varphi_{x}\right), \\
& u_{2}=-z \frac{\partial w}{\partial y}+\psi(z)\left(\frac{\partial w}{\partial y}+\varphi_{y}\right), \\
& u_{3}=w(x, t),
\end{aligned}
$$

where $\mathrm{w}$ is the transverse displacement or displacement in $\mathrm{z}$ direction and $\varphi_{x}, \varphi_{y}$ are the angular displacements in the $\mathrm{x}$ and y directions, respectively. $\psi(z)$ is the shape function as follows: For classical plate theory (CLPT): $\psi(z)=0$

For first order shear deformation theory (FSDT): $\psi(z)=z$ For higher order shear deformation theory (HSDT):

$$
\psi(z)=z-\frac{4 z^{3}}{3 h^{2}}
$$

\subsection{Classical plate theory (CLPT)}

The simplest and the most well-known plate theory is the classical plate theory in which it is assumed that the straight lines which are vertical to the mid-plane will remain straight and vertical to the mid-plane after deformation. In other words, the effects of shear deformation and rotational inertia are not considered in this type of plate theory. On the basis of Eq. (1), the strain-displacement relations appropriate to CLPT can be obtained as:

$$
\begin{aligned}
& \varepsilon_{x x}=\frac{\partial u_{1}}{\partial x}=-z \frac{\partial^{2} w}{\partial x^{2}}, \\
& \varepsilon_{y y}=\frac{\partial u_{2}}{\partial y}=-z \frac{\partial^{2} w}{\partial y^{2}}, \\
& \gamma_{x y}=\frac{\partial u_{1}}{\partial y}+\frac{\partial u_{2}}{\partial x}=-2 z \frac{\partial^{2} w}{\partial x \partial y}, \\
& \gamma_{x z}=\gamma_{y z}=0 .
\end{aligned}
$$

Using the principle of virtual displacement, the equilibrium equation can be expressed for CLPT as:

$$
\frac{\partial^{2} M_{x x}}{\partial x^{2}}+\frac{\partial^{2} M_{y y}}{\partial y^{2}}+2 \frac{\partial^{2} M_{x y}}{\partial x \partial y}=\rho h \frac{\partial^{2} w}{\partial t^{2}},
$$

$$
M=\left\{M_{x x}, M_{y y}, M_{x y}\right\}^{T}=\int_{-h / 2}^{h / 2} z\left\{\sigma_{x x}, \sigma_{y y}, \sigma_{x y}\right\}^{T} d z,
$$

where $M$ is torque, $\rho$ is the mass density and $\sigma$ is axial stress. The governing Eq. (3) can be obtained in terms of displacements as:

$$
\begin{aligned}
& \frac{E h^{3}}{12\left(1-v^{2}\right)}\left(\frac{\partial^{4} w}{\partial x^{4}}+2 v \frac{\partial^{4} w}{\partial x^{2} \partial y^{2}}+\frac{\partial^{4} w}{\partial y^{4}}\right)- \\
& -\frac{E h^{3}}{6(1+v)} \frac{\partial^{4} w}{\partial x^{2} \partial y^{2}}=\rho h \frac{\partial^{2} w}{\partial t^{2}},
\end{aligned}
$$

where $E$ and $v$ are Elastic modulus and poison ration.

\subsection{First order shear deformation theory (FSDT)}

The next plate theory is the first order shear deformation theory in which the effects of shear deformation and rotational inertia are taken into account, so the straight lines will no longer remain vertical to the mid-plane of the plate after deformation. However, it is assumed that the transverse shear stress has a linear distribution along the thickness of the plate. Using Eq. (1), the following strain-displacement relations can be obtained as:

$$
\begin{aligned}
& \varepsilon_{x x}=\frac{\partial u_{1}}{\partial x}=z \frac{\partial \varphi_{x}}{\partial x}, \\
& \varepsilon_{y y}=\frac{\partial u_{2}}{\partial x}=z \frac{\partial \varphi_{y}}{\partial y}, \\
& \gamma_{x y}=\frac{\partial u_{1}}{\partial y}+\frac{\partial u_{2}}{\partial x}=z\left(\frac{\partial \varphi_{x}}{\partial y}+\frac{\partial \varphi_{y}}{\partial x}\right), \\
& \gamma_{x z}=\frac{\partial u_{1}}{\partial z}+\frac{\partial u_{3}}{\partial x}=\frac{\partial w}{\partial x}+\varphi_{x}, \\
& \gamma_{y z}=\frac{\partial u_{2}}{\partial z}+\frac{\partial u_{3}}{\partial y}=\frac{\partial w}{\partial y}+\varphi_{y} .
\end{aligned}
$$

Using the principle of virtual displacement, the equilibrium equations can be expressed for FSDT as:

$$
\begin{aligned}
& \frac{\partial Q_{x x}}{\partial x}+\frac{\partial Q_{y y}}{\partial y}=\rho h \frac{\partial^{2} w}{\partial t^{2}}, \\
& \frac{\partial M_{x x}}{\partial x}+\frac{\partial M_{x y}}{\partial y}-Q_{x x}=\rho h^{3} \frac{\partial^{2} \varphi_{x}}{\partial t^{2}}, \\
& \frac{\partial M_{y y}}{\partial y}+\frac{\partial M_{x y}}{\partial x}-Q_{y y}=\rho h^{3} \frac{\partial^{2} \varphi_{y}}{\partial t^{2}},
\end{aligned}
$$

where $Q=\left\{Q_{x x}, Q_{y y}\right\}^{T}=\int_{-h / 2}^{h / 2}\left\{\sigma_{x z}, \sigma_{y z}\right\}^{T} d z$.

The governing Eq. (6) can be obtained in terms of displacements as: 


$$
\begin{aligned}
& \kappa G h\left(\frac{\partial \varphi_{x}}{\partial x}+\frac{\partial \varphi_{y}}{\partial y}+\frac{\partial^{2} w}{\partial x^{2}}+\frac{\partial^{2} w}{\partial y^{2}}\right)=\rho h \frac{\partial^{2} w}{\partial t^{2}}, \\
& \frac{E h^{3}}{12\left(1-v^{2}\right)}\left(\frac{\partial^{2} \varphi_{x}}{\partial x^{2}}+v \frac{\partial^{2} \varphi_{y}}{\partial x \partial y}\right)+\frac{E h^{3}}{24(1+v)} \times \\
& \times\left(\frac{\partial^{2} \varphi_{x}}{\partial x \partial y}+\frac{\partial^{2} \varphi_{y}}{\partial y^{2}}\right)-\kappa G h\left(\varphi_{x}+\frac{\partial w}{\partial x}\right)=\rho h^{3} \frac{\partial^{2} \varphi_{x}}{\partial t^{2}}, \\
& \frac{E h^{3}}{12\left(1-v^{2}\right)}\left(\frac{\partial^{2} \varphi_{y}}{\partial y^{2}}+v \frac{\partial^{2} \varphi_{x}}{\partial x \partial y}\right)+\frac{E h^{3}}{24(1+v)} \times \\
& \times\left(\frac{\partial^{2} \varphi_{x}}{\partial x^{2}}+\frac{\partial^{2} \varphi_{y}}{\partial x \partial y}\right)-\kappa G h\left(\varphi_{y}+\frac{\partial w}{\partial y}\right)=\rho h^{3} \frac{\partial^{2} \varphi_{y}}{\partial t^{2}} .
\end{aligned}
$$

\subsection{Higher order shear deformation theory (HSDT)}

Another type of plate theory is the third-order shear deformation theory in which the transverse shear stress has a parabolic distribution with respect to the thickness of the plate. Also, there is not any shear correction factor to satisfy the transverse shear stress conditions on the upper and lower layers of the cross-section of the plate. According to Eq. (1), the strain-displacement relations for HSDT can be expressed as:

$$
\begin{aligned}
& \varepsilon_{x x}=\frac{\partial u_{1}}{\partial x}=z \frac{\partial \varphi_{x}}{\partial x}-\frac{4 z^{3}}{3 h^{2}}\left(\frac{\partial \varphi_{x}}{\partial x}+\frac{\partial^{2} w}{\partial x^{2}}\right), \\
& \varepsilon_{y y}=\frac{\partial u_{2}}{\partial x}=z \frac{\partial \varphi_{y}}{\partial y}-\frac{4 z^{3}}{3 h^{2}}\left(\frac{\partial \varphi_{y}}{\partial y}+\frac{\partial^{2} w}{\partial y^{2}}\right), \\
& \gamma_{x y}=\frac{\partial u_{1}}{\partial y}+\frac{\partial u_{2}}{\partial x}=z\left(\frac{\partial \varphi_{x}}{\partial y}+\frac{\partial \varphi_{y}}{\partial x}\right)- \\
& -\frac{4 z^{3}}{3 h^{2}}\left(\frac{\partial \varphi_{x}}{\partial y}+\frac{\partial \varphi_{y}}{\partial x}+2 \frac{\partial^{2} w}{\partial x \partial y}\right), \\
& \gamma_{x z}=\frac{\partial u_{1}}{\partial z}+\frac{\partial u_{3}}{\partial x}=\left(1-\frac{4 z^{2}}{h^{2}}\right)\left(\varphi_{x}+\frac{\partial w}{\partial x}\right), \\
& \gamma_{y z}=\frac{\partial u_{2}}{\partial z}+\frac{\partial u_{3}}{\partial y}=\left(1-\frac{4 z^{2}}{h^{2}}\right)\left(\varphi_{y}+\frac{\partial w}{\partial y}\right)
\end{aligned}
$$

Using the principle of virtual displacement, the equilibrium equations can be expressed for HSDT as:

$$
\begin{aligned}
& \frac{\partial Q_{x x}}{\partial x}+\frac{\partial Q_{y y}}{\partial y}-\frac{4}{h^{2}}\left(\frac{\partial S_{x x}}{\partial x}+\frac{\partial S_{y y}}{\partial y}\right)+ \\
& +\frac{4}{3 h^{2}}\left(\frac{\partial^{2} R_{x x}}{\partial x^{2}}+\frac{\partial^{2} R_{y y}}{\partial y^{2}}+2 \frac{\partial^{2} R}{\partial x \partial y}\right)=\rho h \frac{\partial^{2} w}{\partial t^{2}},
\end{aligned}
$$

$$
\begin{aligned}
& \frac{\partial M_{x x}}{\partial x}+\frac{\partial M_{x y}}{\partial y}-\frac{4}{3 h^{2}}\left(\frac{\partial R_{x x}}{\partial x}+\frac{\partial R_{x y}}{\partial y}\right)-Q_{x x}+\frac{4}{h^{2}} S_{x x}= \\
& =\rho h^{3} \frac{\partial^{2} \varphi_{x}}{\partial t^{2}} \\
& \frac{\partial M_{y y}}{\partial y}+\frac{\partial M_{x y}}{\partial x}-\frac{4}{3 h^{2}}\left(\frac{\partial R_{y y}}{\partial y}+\frac{\partial R_{x y}}{\partial x}\right)- \\
& -Q_{y y}+\frac{4}{h^{2}} S_{y y}=\rho h^{3} \frac{\partial^{2} \varphi_{y}}{\partial t^{2}}
\end{aligned}
$$

where $R=\left\{R_{x x}, R_{y y}, R_{x y}\right\}^{T}=\int_{-h / 2}^{h / 2} z^{3}\left\{\sigma_{x x}, \sigma_{y y}, \sigma_{x y}\right\}^{T} d z$ and

$$
S=\left\{S_{x x}, S_{y y}\right\}^{T}=\int_{-h / 2}^{h / 2} z^{2}\left\{\sigma_{x z}, \sigma_{y z}\right\}^{T} d z
$$

The governing equations of (9) can be obtained in terms of displacements as:

$$
\begin{aligned}
& \frac{8 G h}{15}\left(\frac{\partial \varphi_{x}}{\partial x}+\frac{\partial \varphi_{y}}{\partial y}+\frac{\partial^{2} w}{\partial x^{2}}+\frac{\partial^{2} w}{\partial y^{2}}\right)+\frac{4 E h^{3}}{315\left(1-v^{2}\right)}\left[\frac{\partial^{3} \varphi_{x}}{\partial x^{3}}+\right. \\
& \left.+\frac{\partial^{3} \varphi_{y}}{\partial y^{3}}+v\left(\frac{\partial^{3} \varphi_{x}}{\partial x \partial y^{2}}+\frac{\partial^{3} \varphi_{y}}{\partial x^{2} \partial y}\right)\right]-\frac{4 E h^{3}}{252\left(1-v^{2}\right)}\left(\frac{\partial^{4} w}{\partial x^{4}}+\frac{\partial^{4} w}{\partial y^{4}}+\right. \\
& \left.+2 v \frac{\partial^{4} w}{\partial x^{2} \partial y^{2}}\right)+\frac{4 E h^{3}}{315(1+v)}\left(\frac{\partial^{3} \varphi_{x}}{\partial x^{2} \partial y}+\frac{\partial^{3} \varphi_{y}}{\partial x \partial y^{2}}\right)- \\
& -\frac{E h^{3}}{126(1+v)} \frac{\partial^{4} w}{\partial x^{2} \partial y^{2}}=\rho h \frac{\partial^{2} w}{\partial t^{2}}, \\
& \quad-\frac{8 G h}{15}(10-\mathrm{a}) \\
& \quad \frac{17 E h^{3}}{315\left(1-v^{2}\right)}\left(\frac{\varphi^{2} \varphi_{y}}{\partial y^{2}}+v \frac{\partial^{2} \varphi_{x}}{\partial x \partial y}\right)=\frac{4 E h^{3}}{315\left(1-v^{2}\right)} \times \\
& \quad \times\left(\frac{\partial^{3} w}{\partial y^{3}}+v \frac{\partial^{2} \varphi_{x}}{\partial x^{2} \partial y}\right)+\frac{17 E t^{2}}{630(1+v)}\left(\frac{\partial^{2} \varphi_{x}}{\partial x^{2}}+\frac{\partial^{2} \varphi_{y}}{\partial x \partial y}\right)- \\
& -\frac{4 E h^{3}}{315(1+v)} \frac{\partial^{3} w}{\partial x^{2} \partial y}-\frac{8 G h}{15}\left(\varphi_{y}+\frac{\partial w}{\partial y}\right)= \\
& \quad
\end{aligned}
$$

\section{Nonlocal plate theories for free vibration of SLGSs}

\subsection{Review of Eringen's nonlocal elasticity}

The theory of nonlocal elasticity was first considered by Eringen in the 1970's [25]. This concept is inherent in solid state physics where the nonlocal attractions of atoms are prevalent Eringen. In contrast to the classical elasticity, in the nonlocal model the stress at a reference point $\mathrm{x}$ in an elastic body depends not only on the strains at $\mathrm{x}$, but also on strains at all other points of the body [25]. According to the nonlocal elasticity theory, this fact was attributed to the 
atomic theory of lattice dynamics and experimental measurements of phonon dispersion [26].

For homogenous and isotropic elastic continuum, the linear nonlocal elasticity theory can be expressed as the following set of equations [26]:

$$
\begin{aligned}
& \sigma_{k l, k}+\rho\left(f_{l}-\ddot{u}_{l}\right)=0, \\
& \sigma_{k l}(x)=\int_{V} \alpha\left(\left|x-x^{\prime}\right|, \tau\right) \sigma_{k l}^{c}\left(x^{\prime}\right) d V, \\
& \sigma_{k l}^{c}\left(x^{\prime}\right)=L_{1} e_{r r}\left(x^{\prime}\right) \delta_{k l}+2 L_{2} e_{k l}\left(x^{\prime}\right), \\
& e_{k l}\left(x^{\prime}\right)=\frac{1}{2}\left(\frac{d u_{k}\left(x^{\prime}\right)}{d x_{l}^{\prime}}+\frac{d u_{l}\left(x^{\prime}\right)}{d x_{k}^{\prime}}\right) .
\end{aligned}
$$

Where equation (11-a) is the equilibrium relation in which $\sigma_{k l, l}, \rho, f_{l}$ and $\mathrm{u}_{1}$ are the nonlocal stress tensor, mass density, body force density and displacement vector at a reference point $x$ in the body, respectively. Eq. (11-b) is the relation between local $\left(\sigma_{k l}^{c}\right)$ and nonlocal $\left(\sigma_{k l, l}\right)$ stress tensors using the nonlocal modulus $\left(\alpha\left(\left|x-x^{\prime}\right|, \tau\right)\right)$. Finally, equation (11-c) and (11-d) are the classical constitutive stress-strain and strain-displacement relationships, respectively. $L_{1}$ and $L_{2}$ are the Lame constants.

Eringen [24] made certain assumptions to simplify equation (11-b) to a partial differential equation form as:

$$
\left(1-\tau^{2} l^{2} \nabla^{2}\right) t_{k l}(x)=\sigma_{k l}(x), \tau=e_{0} \frac{a}{l} .
$$

Where $t_{k l}=\sigma_{k l, l}, a / l$ is the characteristic length ratio and $e_{0}$ is the nonlocal constant which are appropriate to the material.

\subsection{Application of elastic medium and nonlocal elasticity on beam theories}

The nanobeams analyzed in this work are assumed to be embedded in an elastic medium. The elastic surrounding is simulated using Pasternak foundation model [30], which considers both normal pressure and transverse shear stress. So the loading corresponding to this type of foundation model yields as [30]:

$$
Q_{\text {Pasternak }}=K_{w} w-K_{s} \frac{\partial^{2} w}{\partial x^{2}}
$$

where $K_{w}$ is the Winkler modulus parameter corresponding to normal pressure, and $K_{s}$ is the Pasternak modulus parameter relevant to transverse shear stress.

By neglecting the shear deformation effects $\left(K_{s}=0\right)$, the foundation model is reduced to Winkler one [29], which represents the normal pressure of elastic medium as a series of closely spaced, vertical linear elastic springs. Thereupon, the loading corresponding to this type of foundation can be expressed as [21].

$$
Q_{\text {Winkler }}=K_{w} w
$$

In this work, the buckling behavior of nanobeams surrounding in an elastic medium is investigated using both above types of foundation models based on various nonlocal beam theories.

\subsubsection{Euler-Bernoulli beam theory}

By adding the elastic medium terms to the governing equation of EBT, we will have:

$$
-E I \frac{\partial^{4} w}{\partial x^{4}}+K_{s} \frac{\partial^{2} w}{\partial x^{2}}-K_{w} w=\rho A \frac{\partial^{2} w}{\partial t^{2}}-\rho I \frac{\partial^{4} w}{\partial x^{2} \partial t^{2}}
$$

Using Eq. (13), the only constitutive relation for nonlocal model of EBT with elastic medium is obtained as:

$$
\begin{aligned}
& -\left(\mu K_{s}+E I\right) \frac{\partial^{4} w}{\partial x^{4}}+\left(\mu K_{w}+K_{s}\right) \frac{\partial^{2} w}{\partial x^{2}}-K_{w} w= \\
& =\rho A \frac{\partial^{2} w}{\partial t^{2}}-(\mu \rho A+\rho I) \frac{\partial^{4} w}{\partial x^{2} \partial t^{2}}+\mu \rho I \frac{\partial^{6} w}{\partial x^{4} \partial t^{2}} .
\end{aligned}
$$

\section{2. 2. Timoshenko beam theory}

Adding the elastic medium terms to the governing equations of TBT yields:

$$
\begin{aligned}
& \left(\kappa G A+K_{s}\right) \frac{\partial^{2} w}{\partial x^{2}}-K_{w} w+\kappa G A \frac{\partial \varphi}{\partial x}=\rho A \frac{\partial^{2} w}{\partial t^{2}} \\
& -\kappa G A \frac{\partial w}{\partial x}+E I \frac{\partial^{2} \varphi}{\partial x^{2}}-\kappa G A \varphi=\rho I \frac{\partial^{2} \varphi}{\partial t^{2}} .
\end{aligned}
$$

Using equation (13), the constitutive relations for nonlocal model of TBT with elastic medium can be expressed as:

$$
\begin{aligned}
& -\mu K_{s} \frac{\partial^{4} w}{\partial x^{4}}+\left(\mu K_{w}+K_{s}+\kappa G A\right) \frac{\partial^{2} w}{\partial x^{2}}-K_{w} w+ \\
& +\kappa G A \frac{\partial \varphi}{\partial x}=\rho A \frac{\partial^{2} w}{\partial t^{2}}-\mu \rho A \frac{\partial^{4} w}{\partial x^{2} \partial t^{2}} \\
& -\kappa G A \frac{\partial w}{\partial x}+E I \frac{\partial^{2} \varphi}{\partial x^{2}}-\kappa G A \varphi=\rho I \frac{\partial^{2} \varphi}{\partial t^{2}}- \\
& -\mu \rho I \frac{\partial^{4} \varphi}{\partial x^{2} \partial t^{2}} .
\end{aligned}
$$

\subsubsection{Reddy beam theory}

By adding the elastic medium terms to the governing equations of RBT, we will have:

$$
\begin{aligned}
& -\frac{E I}{21} \frac{\partial^{4} w}{\partial x^{4}}+\left(\frac{8 G A}{15}+K_{s}\right) \frac{\partial^{2} w}{\partial x^{2}}+\frac{16 E I}{105} \frac{\partial^{3} \varphi}{\partial x^{3}}+ \\
& +\frac{8 G A}{15} \frac{\partial \varphi}{\partial x}-K_{w} w=\rho A \frac{\partial^{2} w}{\partial t^{2}} .
\end{aligned}
$$




$$
\begin{aligned}
& -\frac{16 E I}{105} \frac{\partial^{3} w}{\partial x^{3}}-\frac{8 G A}{15} \frac{\partial w}{\partial x}+ \\
& +\frac{68 E I}{105} \frac{\partial^{2} \varphi}{\partial x^{2}}-\frac{8 G A}{15} \varphi=\rho I \frac{\partial^{2} \varphi}{\partial t^{2}} .
\end{aligned}
$$

Using equation (13), the constitutive relations for nonlocal model of RBT with elastic medium are obtained as:

$$
\begin{aligned}
& -\left(\mu K_{s}+\frac{E I}{21}\right) \frac{\partial^{4} w}{\partial x^{4}}+\left(\mu K_{w}+K_{s}+\frac{8 G A}{15}\right) \frac{\partial^{2} w}{\partial x^{2}}- \\
& -K_{w} w+\frac{16 E I}{105} \frac{\partial^{3} \varphi}{\partial x^{3}}+\frac{8 G A}{15} \frac{\partial \varphi}{\partial x}= \\
& =\rho A \frac{\partial^{2} w}{\partial t^{2}}-\mu \rho A \frac{\partial^{4} w}{\partial x^{2} \partial t^{2}}, \\
& -\frac{16 E I}{105} \frac{\partial^{3} w}{\partial x^{3}}-\frac{8 G A}{15} \frac{\partial w}{\partial x}+\frac{68 E I}{105} \frac{\partial^{2} \varphi}{\partial x^{2}}-\frac{8 G A}{15} \varphi= \\
& =\rho I \frac{\partial^{2} \varphi}{\partial t^{2}}-\mu \rho I \frac{\partial^{4} \varphi}{\partial x^{2} \partial t^{2}} .
\end{aligned}
$$

\section{2. 4. Levinson beam theory}

Adding the elastic medium terms to the governing equations of LBT yields:

$$
\begin{aligned}
& \left(\frac{2 G A}{3}+K_{s}\right) \frac{\partial^{2} w}{\partial x^{2}}-K_{w} w+\frac{2 G A}{3} \frac{\partial \varphi}{\partial x}=\rho A \frac{\partial^{2} w}{\partial t^{2}}, \\
& -\frac{E I}{5} \frac{\partial^{3} w}{\partial x^{3}}-\frac{2 G A}{3} \frac{\partial w}{\partial x}+\frac{4 E I}{5} \frac{\partial^{2} \varphi}{\partial x^{2}}-\frac{2 G A}{3} \varphi= \\
& =\rho I \frac{\partial^{2} \varphi}{\partial t^{2}} .
\end{aligned}
$$

Using Eq. (13), the constitutive relations for nonlocal model of LBT with elastic medium can be expressed as:

$$
\begin{aligned}
& -\mu K_{s} \frac{\partial^{4} w}{\partial x^{4}}+\left(\mu K_{w}+K_{s}+\frac{2 G A}{3}\right) \frac{\partial^{2} w}{\partial x^{2}}- \\
& -K_{w} w+\frac{2 G A}{3} \frac{\partial \varphi}{\partial x}= \\
& =\rho A \frac{\partial^{2} w}{\partial t^{2}}-\mu \rho A \frac{\partial^{4} w}{\partial x^{2} \partial t^{2}}, \\
& -\frac{E I}{5} \frac{\partial^{3} w}{\partial x^{3}}-\frac{2 G A}{3} \frac{\partial w}{\partial x}+\frac{4 E I}{5} \frac{\partial^{2} \varphi}{\partial x^{2}}-\frac{2 G A}{3} \varphi= \\
& =\rho I \frac{\partial^{2} \varphi}{\partial t^{2}}-\mu \rho I \frac{\partial^{4} w}{\partial x^{2} \partial t^{2}} .
\end{aligned}
$$

It is worth to mention that by removing the surrounded elastic medium effects from the governing equations corresponding to each beam theory, they reduce to the conventional nonlocal beam theories presented by Reddy [27].

\section{Analytical solution for simply supported nanobeams}

4.1. Explicit formulas for natural frequencies

In this section, exact solutions of free vibration of nanobeams embedded in an elastic medium are developed. Explicit formulas are proposed to obtain the natural frequencies corresponding to each nonlocal beam theory. The simply supported boundary conditions can be expressed as:

$$
\begin{aligned}
& w(0)=w(L)=0, \\
& M(0)=M(L)=0 .
\end{aligned}
$$

The components of displacement $\mathrm{w}$ and $\varphi$ can be considered in the following generalized form which satisfies the boundary conditions:

$$
\begin{aligned}
& w(x, t)=\sum_{m=1}^{\infty} W_{m} \sin \left(\frac{m \pi x}{L}\right) e^{i \omega_{m} t}, \\
& \varphi(x, t)=\sum_{m=1}^{\infty} \phi_{m} \cos \left(\frac{m \pi x}{L}\right) e^{i \omega_{m} t} .
\end{aligned}
$$

Substituting Eqs. (25) in the constitutive relations of different nonlocal beam theories and solving the resulting eigenvalue problem, the natural frequencies of nanobeams embedded in an elastic medium can be obtained.

For Euler-Bernoulli beam theory, the natural frequencies can be expressed as:

$$
\begin{aligned}
& \omega_{E B T}^{2}=\frac{\left(m^{4} \pi^{4} \mu K_{s}+m^{4} \pi^{4} E I+m^{2} \pi^{2} \mu K_{w} L^{2}+\right.}{\left(m^{2} \pi^{2} \rho A \mu L^{2}+\rho A L^{4}\right)} \\
& \frac{\left.+m^{2} \pi^{2} K_{s} L^{2}+K_{w} L^{4}\right)}{\left(m^{2} \pi^{2} \rho A \mu L^{2}+\rho A L^{4}\right)} .
\end{aligned}
$$

For Timoshenko beam theory, the natural frequencies can be obtained as follows:

$$
\begin{aligned}
& \omega_{T B T}^{2}=\left(m^{6} \pi^{6} \mu K_{s} E I+m^{4} \pi^{4} \mu K_{s} \kappa G A L^{2}+\right. \\
& +m^{4} \pi^{4} \mu K_{w} E I L^{2}+m^{2} \pi^{2} \mu K_{w} \kappa G A L^{4}+ \\
& +m^{4} \pi^{4} K_{s} E I L^{2}+m^{2} \pi^{2} K_{s} \kappa G A L^{4}+ \\
& +m^{4} \pi^{4} \kappa G A E I L^{2}+m^{2} \pi^{2} K_{w} E I L^{4}+ \\
& \left.+K_{w} \kappa G A L^{6}\right) .
\end{aligned}
$$

For Reddy beam theory, the natural frequencies can be expressed as:

$$
\begin{aligned}
& \omega_{R B T}^{2}=\frac{1}{5}\left(85 m^{6} \pi^{6} \mu K_{s} E I+70 m^{4} \pi^{4} \mu K_{s} G A L^{2}+\right. \\
& +m^{6} \pi^{6} E^{2} I^{2}+70 m^{4} \pi^{4} E I G A L^{2}+85 m^{4} \pi^{4} \mu K_{w} E I L^{2}+ \\
& +70 m^{2} \pi^{2} \mu K_{w} G A L^{4}+85 m^{4} \pi^{4} K_{s} E I L^{2}+ \\
& \frac{+70 m^{2} \pi^{2} K_{s} G A L^{4}+85 m^{2} \pi^{2} K_{w} E I L^{4}+}{+\left(17 m^{2} \pi^{2} \rho A E I L^{4}+14 \rho G A^{2} L^{6}+\right.} \\
& \frac{\left.+70 K_{w} G A L^{6}\right)}{\left.+17 m^{4} \pi^{4} \mu \rho A E I L^{2}+14 m^{2} \pi^{2} \mu \rho G A^{2} L^{4}\right)} .
\end{aligned}
$$


For Levinson beam theory, the natural frequencies can be obtained as follows:

$$
\begin{aligned}
& \omega_{L B T}^{2}=\left(6 m^{6} \pi^{6} \mu K_{s} E I+5 m^{4} \pi^{4} \mu K_{s} G A L^{2}+\right. \\
& +6 m^{4} \pi^{4} \mu K_{w} E I L^{2}+5 m^{2} \pi^{2} \mu K_{w} G A L^{4}+ \\
& +6 m^{4} \pi^{4} K_{s} E I L^{2}+5 m^{2} \pi^{2} K_{s} G A L^{4}+ \\
& +5 m^{4} \pi^{4} G A E I L^{2}+6 m^{2} \pi^{2} K_{w} E I L^{4}+ \\
& \left.+5 K_{w} G A L^{6}\right) /\left(6 m^{2} \pi^{2} \rho A E I L^{4}+5 \rho G A^{2} L^{6}+\right. \\
& \left.+6 m^{4} \pi^{4} \rho A \mu E I L^{2}+5 m^{2} \pi^{2} \rho G A^{2} L^{4}\right) .
\end{aligned}
$$

\subsection{Numerical results and discussion}

Here the numerical results are presented for the developed analytical solution in the previous section. The fol- lowing properties are taken for the nanobeams which are used by Reddy [27].

$$
E=30 \times 10^{6} \mathrm{~N} / \mathrm{m}^{2}, v=0.3, \rho=1 .
$$

It is assumed that $h=b=1 \mathrm{~nm}$ and $L$ varies from $\frac{L}{h}=10$ to 50

The non-dimensional natural frequencies corresponding to the first three modes of the nanotubes are given in Tables 1-3. It's found from the results that with increasing the value of nonlocal parameter the values of natural frequencies decrease, especially for lower aspect ratios. It im-

\begin{tabular}{|c|c|c|c|c|c|c|c|c|c|}
\hline$L / h$ & $\mu$ & $\begin{array}{c}E B T \\
K_{w}=0 \\
K_{s}=0\end{array}$ & $\begin{array}{c}E B T \\
K_{w}=20 \\
K_{s}=2\end{array}$ & $\begin{array}{c}T B T \\
K_{w}=0 \\
K_{s}=0\end{array}$ & $\begin{array}{c}T B T \\
K_{w}=20 \\
K_{s}=2\end{array}$ & $\begin{array}{c}R B T \\
K_{w}=0 \\
K_{s}=0\end{array}$ & $\begin{array}{c}R B T \\
K_{w}=20 \\
K_{s}=2\end{array}$ & $\begin{array}{c}L B T \\
K_{w}=0 \\
K_{s}=0\end{array}$ & $\begin{array}{c}L B T \\
K_{w}=20 \\
K_{s}=2\end{array}$ \\
\hline \multirow{5}{*}{10} & 0 & 9.8696 & 9.8737 & 9.7519 & 9.7561 & 9.7520 & 9.7561 & 9.7713 & 9.7756 \\
\hline & 0.5 & 9.6347 & 9.6389 & 9.5199 & 9.5241 & 9.5199 & 9.5241 & 9.5387 & 9.5432 \\
\hline & 1 & 9.4159 & 9.4202 & 9.3036 & 9.3080 & 9.3036 & 9.3080 & 9.3220 & 9.3266 \\
\hline & 1.5 & 9.2113 & 9.2157 & 9.1015 & 9.1059 & 9.1015 & 9.1059 & 9.1195 & 9.1241 \\
\hline & 2 & 9.0195 & 9.0240 & 8.9119 & 8.9165 & 8.9120 & 8.9165 & 8.9296 & 8.9343 \\
\hline \multirow{5}{*}{20} & 0 & 9.8696 & 9.9344 & 9.8398 & 9.9048 & 9.8398 & 9.9048 & 9.8400 & 9.9050 \\
\hline & 0.5 & 9.8093 & 9.8745 & 9.7796 & 9.8450 & 9.7796 & 9.8450 & 9.7798 & 9.8452 \\
\hline & 1 & 9.7501 & 9.8156 & 9.7206 & 9.7864 & 9.7206 & 9.7864 & 9.7208 & 9.7866 \\
\hline & 1.5 & 9.6919 & 9.7579 & 9.6626 & 9.7288 & 9.6626 & 9.7288 & 9.6628 & 9.7291 \\
\hline & 2 & 9.6347 & 9.7011 & 9.6056 & 9.6722 & 9.6056 & 9.6722 & 9.6059 & 9.6725 \\
\hline \multirow{5}{*}{50} & 0 & 9.8696 & 12.1420 & 9.8648 & 12.1381 & 9.8648 & 12.1381 & 9.8648 & 12.1381 \\
\hline & 0.5 & 9.8599 & 12.1341 & 9.8551 & 12.1302 & 9.8551 & 12.1302 & 9.8552 & 12.1303 \\
\hline & 1 & 9.8502 & 12.1262 & 9.8454 & 12.1224 & 9.8454 & 12.1224 & 9.8454 & 12.1225 \\
\hline & 1.5 & 9.8405 & 12.1184 & 9.8357 & 12.1145 & 9.8357 & 12.1145 & 9.8357 & 12.1146 \\
\hline & 2 & 9.8309 & 12.1106 & 9.8261 & 12.1067 & 9.8261 & 12.1067 & 9.8261 & 12.1068 \\
\hline
\end{tabular}
plies that the nonlocality effect is more significant for the shorter nanobeams and this effect tends to decrease the stiffness of the nanobeam. Moreover, the small-size effect is

\begin{tabular}{|c|c|c|c|c|c|c|c|c|c|}
\hline$L / h$ & $\mu$ & $\begin{array}{c}E B T \\
K_{w}=0 \\
K_{s}=0\end{array}$ & 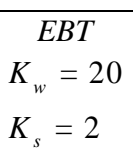 & $\begin{array}{c}T B T \\
K_{w}=0 \\
K_{s}=0\end{array}$ & $\begin{array}{c}\quad T B T \\
K_{w}=20 \\
K_{s}=2\end{array}$ & $\begin{array}{c}R B T \\
K_{w}=0 \\
K_{s}=0\end{array}$ & $\begin{array}{c}\quad R B T \\
K_{w}=20 \\
K_{s}=2\end{array}$ & $\begin{array}{c}L B T \\
K_{w}=0 \\
K_{s}=0\end{array}$ & $\begin{array}{c}\quad L B T \\
K_{w}=20 \\
K_{s}=2\end{array}$ \\
\hline \multirow{5}{*}{10} & 0 & 39.4784 & 39.4795 & 37.6906 & 37.6917 & 37.6925 & 37.6936 & 37.7615 & 37.7626 \\
\hline & 0.5 & 36.0779 & 36.0791 & 34.4441 & 34.4453 & 34.4459 & 34.4471 & 34.5152 & 34.5164 \\
\hline & 1 & 33.4277 & 33.4289 & 31.9139 & 31.9152 & 31.9155 & 31.9168 & 31.9848 & 31.9861 \\
\hline & 1.5 & 31.2870 & 31.2883 & 29.8702 & 29.8716 & 29.8717 & 29.8731 & 28.9410 & 29.9424 \\
\hline & 2 & 29.5111 & 29.5125 & 28.1747 & 28.1762 & 28.1761 & 28.1776 & 28.2454 & 28.2469 \\
\hline \multirow{5}{*}{20} & 0 & 39.4784 & 39.4948 & 39.0077 & 39.0243 & 39.0079 & 39.0244 & 39.0088 & 39.0253 \\
\hline & 0.5 & 38.5390 & 38.5557 & 38.0795 & 38.0964 & 38.0796 & 38.0966 & 38.0805 & 38.0975 \\
\hline & 1 & 37.6635 & 37.6807 & 37.2145 & 37.2318 & 37.2146 & 37.2320 & 37.2155 & 37.2329 \\
\hline & 1.5 & 36.8452 & 36.8627 & 36.4059 & 36.4236 & 36.4060 & 36.4237 & 36.4069 & 36.4246 \\
\hline & 2 & 36.0779 & 36.0958 & 35.6478 & 35.6659 & 35.6479 & 35.6660 & 35.6489 & 35.6669 \\
\hline \multirow{5}{*}{50} & 0 & 39.4784 & 40.1077 & 39.4020 & 40.0324 & 39.4020 & 40.0324 & 39.4021 & 40.0325 \\
\hline & 0.5 & 39.3235 & 39.9552 & 39.2473 & 39.8802 & 39.2473 & 39.8802 & 39.2475 & 39.8804 \\
\hline & 1 & 39.1704 & 39.8045 & 39.0945 & 39.7298 & 39.0945 & 39.7298 & 39.0947 & 39.7300 \\
\hline & 1.5 & 39.0190 & 39.6555 & 38.9434 & 39.5812 & 38.9434 & 39.5812 & 38.9435 & 39.5814 \\
\hline & 2 & 38.8694 & 39.5083 & 38.7941 & 39.4343 & 38.7941 & 39.4343 & 38.7943 & 39.4346 \\
\hline
\end{tabular}
more prominent at higher mode numbers.

Table 1

Non-dimensional natural frequencies $\left(\omega \times L^{2} \sqrt{\frac{\rho A}{E I}}\right)$ of the first mode

Table 2

Non-dimensional natural frequencies $\left(\omega \times L^{2} \sqrt{\frac{\rho A}{E I}}\right)$ of the second mode 
Non-dimensional natural frequencies $\left(\omega \times L^{2} \sqrt{\frac{\rho A}{E I}}\right)$ of the third mode

\begin{tabular}{|c|c|c|c|c|c|c|c|c|c|}
\hline$L / h$ & $\mu$ & $\begin{array}{c}E B T \\
K_{w}=0 \\
K_{s}=0\end{array}$ & $\begin{array}{c}E B T \\
K_{w}=20 \\
K_{s}=2\end{array}$ & $\begin{array}{c}T B T \\
K_{w}=0 \\
K_{s}=0\end{array}$ & $\begin{array}{c}T B T \\
K_{w}=20 \\
K_{s}=2\end{array}$ & $\begin{array}{c}R B T \\
K_{w}=0 \\
K_{s}=0\end{array}$ & $\begin{array}{c}R B T \\
K_{w}=20 \\
K_{s}=2\end{array}$ & $\begin{array}{c}L B T \\
K_{w}=0 \\
K_{s}=0\end{array}$ & $\begin{array}{c}L B T \\
K_{w}=20 \\
K_{s}=2\end{array}$ \\
\hline \multirow{5}{*}{10} & 0 & 88.8264 & 88.8269 & 80.4687 & 80.4693 & 80.4875 & 80.4880 & 80.6519 & 80.7705 \\
\hline & 0.5 & 73.9161 & 73.9166 & 66.9613 & 66.9619 & 66.9769 & 66.9775 & 67.1418 & 67.0963 \\
\hline & 1 & 64.6414 & 64.6421 & 58.5593 & 58.5600 & 58.5729 & 58.5737 & 58.6917 & 58.8105 \\
\hline & 1.5 & 58.1622 & 58.1630 & 52.6897 & 52.6906 & 52.7020 & 52.7028 & 52.8208 & 52.8216 \\
\hline & 2 & 53.3078 & 53.3087 & 48.2921 & 48.2930 & 48.3033 & 48.3042 & 48.4221 & 48.4230 \\
\hline \multirow{6}{*}{20} & 0 & 88.8264 & 88.8338 & 86.4953 & 86.5029 & 86.4968 & 86.5043 & 86.5000 & 86.5075 \\
\hline & 0.5 & 84.2711 & 84.2789 & 82.0595 & 82.0675 & 82.0609 & 82.0689 & 82.0643 & 82.0723 \\
\hline & 1 & 80.3517 & 80.3598 & 78.2429 & 78.2513 & 78.2443 & 78.2526 & 78.2477 & 78.2560 \\
\hline & 1.5 & 76.9327 & 76.9412 & 74.9137 & 74.9224 & 74.9150 & 74.9237 & 74.9185 & 74.9271 \\
\hline & 2 & 73.9161 & 73.9249 & 71.9762 & 71.9853 & 71.9774 & 71.9865 & 71.9808 & 71.9899 \\
\hline & \multicolumn{9}{|c|}{ Table 3 continued } \\
\hline \multirow{5}{*}{50} & 0 & 88.8264 & 89.1084 & 88.4408 & 88.7240 & 88.4408 & 88.7240 & 88.4411 & 88.7243 \\
\hline & 0.5 & 88.0478 & 88.3323 & 87.6655 & 87.9512 & 87.6655 & 87.9513 & 87.6659 & 87.9517 \\
\hline & 1 & 87.2893 & 87.5762 & 86.9103 & 87.1985 & 86.9103 & 87.1985 & 86.9107 & 87.1989 \\
\hline & 1.5 & 86.5500 & 86.8394 & 86.1742 & 86.4649 & 86.1742 & 86.4649 & 86.1746 & 86.4653 \\
\hline & 2 & 85.8292 & 86.1210 & 85.4566 & 85.7496 & 85.4566 & 85.7497 & 85.4569 & 85.7501 \\
\hline
\end{tabular}

Also, it can be seen that by considering the influence of transverse shear strain using $T B T, R B T$, and $L B T$, the values of natural frequencies will be reduced for all values of nonlocal parameter specifically for higher modes. Furthermore, the difference between $T B T$ and $R B T$ is so negligible for the first mode, but it is relatively more sensible for higher modes and lower aspect ratios.

By incorporating the elastic foundation, the nondimensional natural frequencies increase for all values of nonlocal parameter which indicates that surrounding in an elastic medium makes the nanobeams stiffer and at the first mode, this increase of stiffness is more prominent for higher values of aspect ratio. However, for higher modes, the effect of elastic medium tends to be independent from the value of aspect ratio. Fig. 1 depicts this pattern in more sensible way, in which the slope of variation of non-dimensional natural frequency with the value of aspect ratio decreases for higher mode numbers.

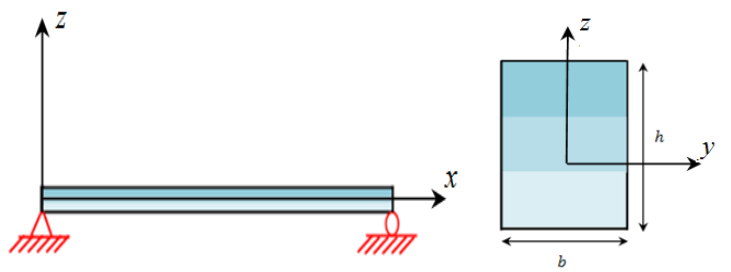

Fig. 1 Simply supported straight uniform plate with rectangular cross section and its coordinate system

It should be noted that the results depicted in Fig. 2 and the other Figures are corresponding to Reddy beam theory, and because there is not any consequential difference between the behaviors of various beam theories, the results of other beam theories are not given for brevity. The Winkler modulus parameter effect on the value of natural frequency of nanobeams with different aspect ratios is plotted in Fig. 3. It is assumed that $\mu=1$ and the nanobeam is represented as Winkler foundation model $\left(K_{s}=0\right)$. The Winkler modulus parameter is taken in the range of $0-400$ used by Liew et al. [31]. This range of $K_{w}$ includes the interval of soft elastic medium to a very stiff one. It can be observed that the effect of $K_{w}$ is more considerable for higher values of aspect ratio.

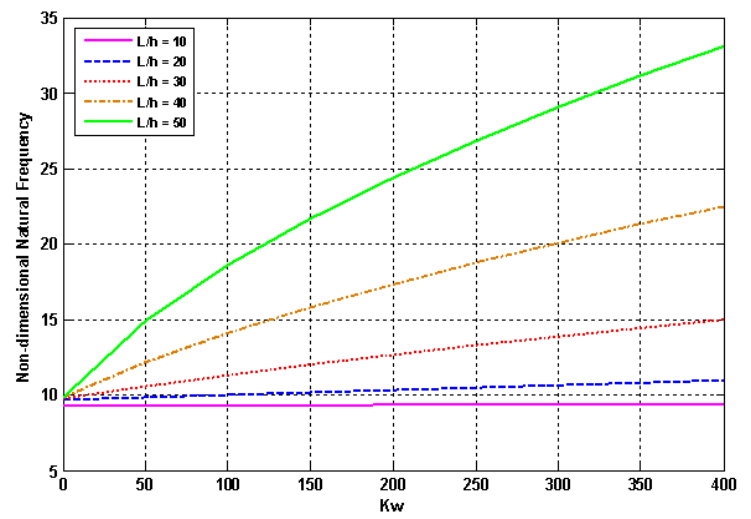

Fig. 2 Variation of non-dimensional natural frequency with Winkler modulus parameter corresponding to different values of aspect ratio $(\mu=1)$

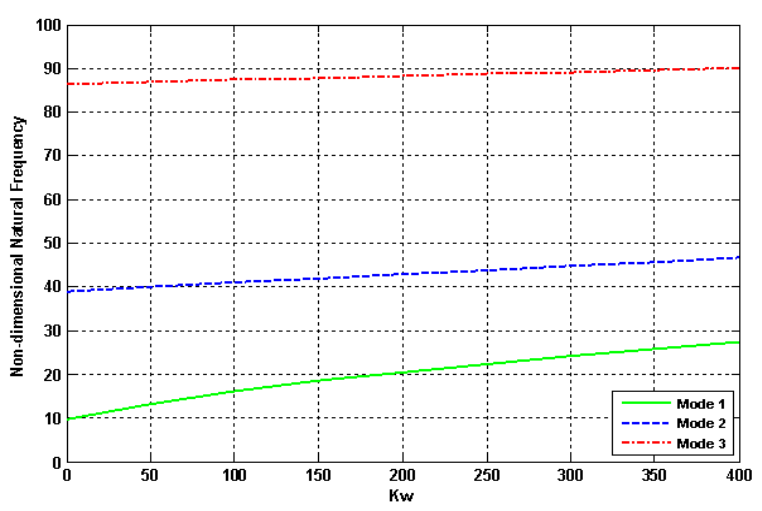

Fig. 3 Variation of non-dimensional natural frequency with Winkler modulus parameter corresponding to different mode numbers $(\mu=1, L / h=45)$ 
To investigate the effect of Pasternak modulus parameter on the vibrational behavior of nanobeams, the variation of non-dimensional natural frequency of nanobeam with the value of Pasternak modulus parameter is depicted in Fig. 4 corresponding to different nonlocal parameters. The surrounding elastic medium is simulated as Pasternak foundation model with $K_{w}=100$. Fig. 4 -a shows this effect for $L / h=10$ and Fig. 4-b shows this effect corresponding to $L / h=50$. It can be found that as the aspect ratio of nanobeams increases $K_{s}$ has more significant influence on the value of critical buckling load.

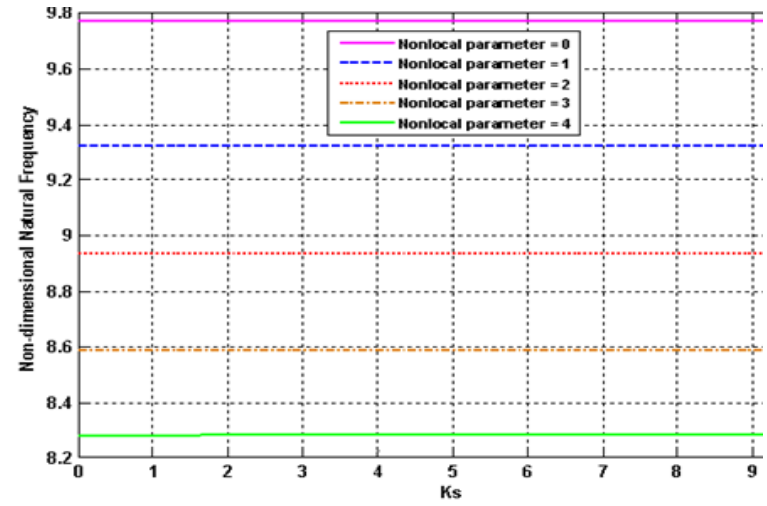

a

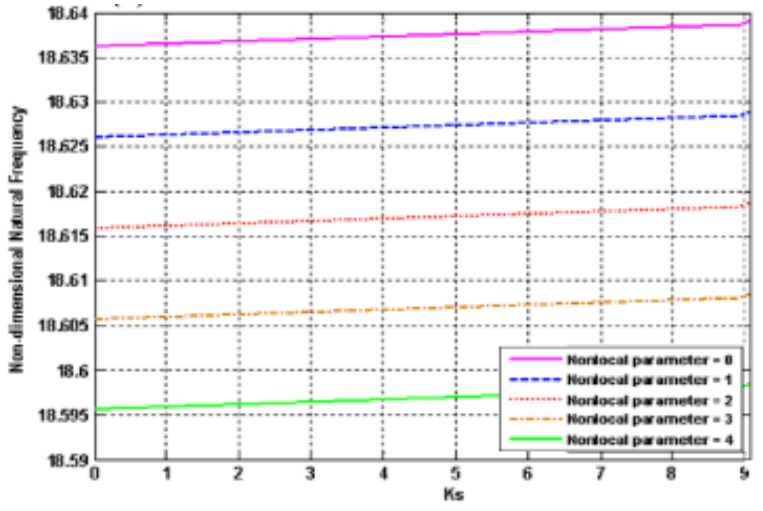

$\mathrm{b}$

Fig. 4 Effect of Pasternak modulus parameter on non-dimensional natural frequency for different values of nonlocal parameter: (a) $K_{w}=100, L / h=10$,

$K_{w}=100, L / h=50$

The mode-shapes for the transverse displacement $\mathrm{w}$ and angular displacement $\varphi$ corresponding to various Winkler modulus parameter and nonlocal parameter are shown in Fig. 5. In all cases, the configuration of modeshapes are obtained at $w_{\max }=1.0$. There is not any significant difference between mode-shapes for various aspect ratios, so they are described just corresponding to $L / h=10$ for brevity. It can be observed that the mode-shapes relevant to simply supported-simply supported boundary conditions have not any sensible dependency to the both values of Winkler modulus parameter and nonlocal parameter.

Fig. 6 indicates the difference between the natural frequencies obtained by Winkler foundation model and the Pasternak one. To this end, the variation of non-dimensional natural frequencies with the value of aspect ratio is plotted corresponding to both foundation models. It is assumed that $\mu=1$ throughout the range of aspect ratio and $K_{w}=20$, $K_{s}=0$ for Winkler foundation model and $K_{w}=20, K_{s}=2$ for Pasternak foundation model.
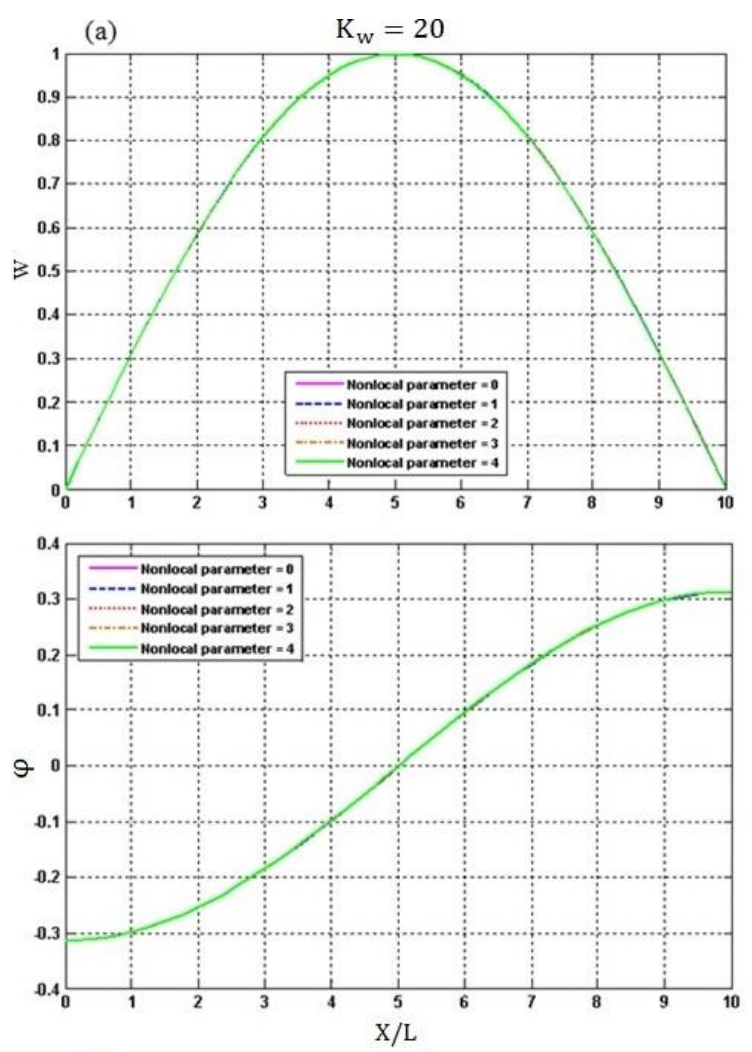

(b) $\mu=1$
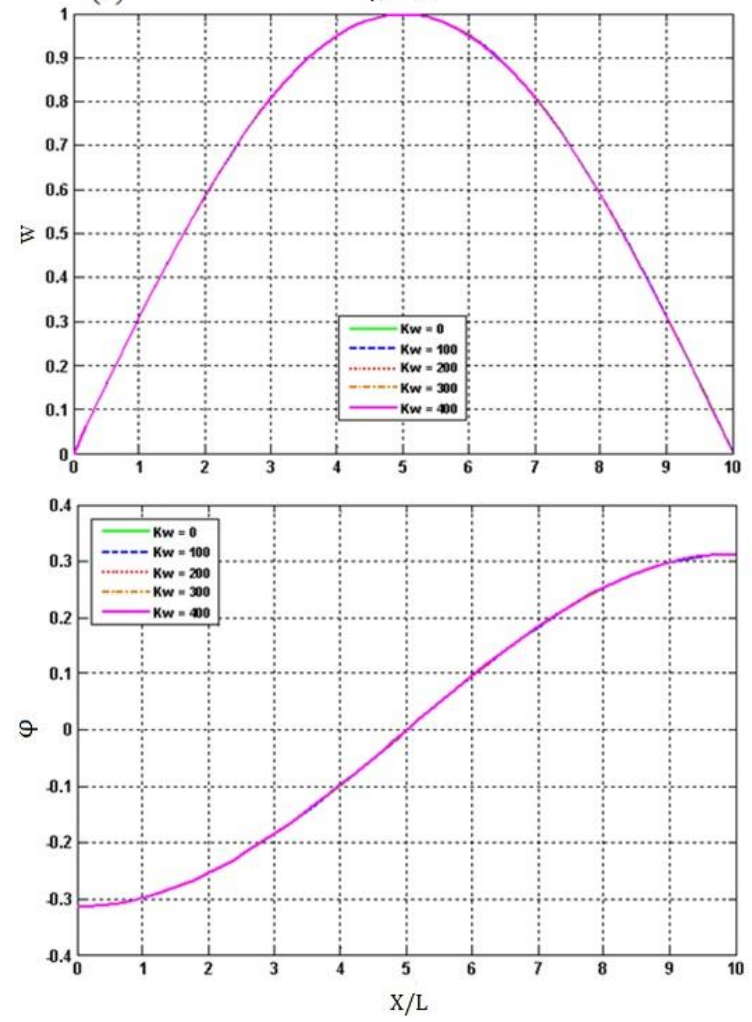

Fig. 5 Effects of nonlocal parameter and Winkler modulus parameter on the mode-shapes of transverse and angular displacements with: (a) $K_{w}=20$; (b) $\mu=1$

As shown in the Fig. 6, for each aspect ratio, the calculated natural frequency with the Winkler-based models 
is lower than the natural frequency calculated by the Pasternak models. More, as the value of aspect ratio increases the difference between two foundation models increases too. This reveals that alike the observation from Fig. 4, Pasternak modulus parameter has more significant influence at higher values of aspect ratio.

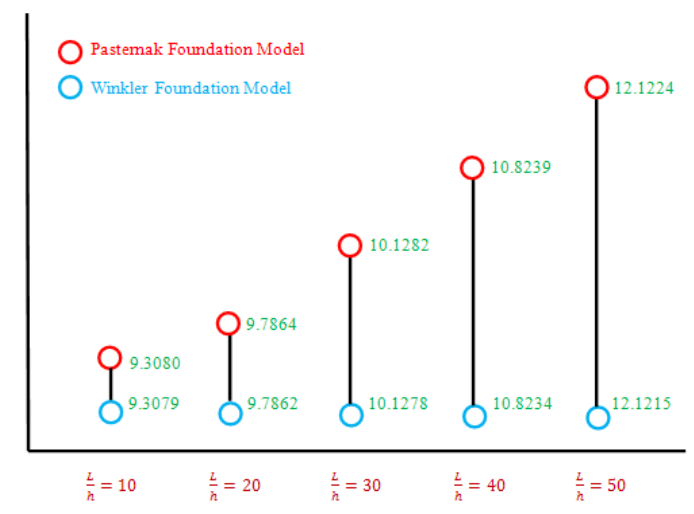

Fig. 6 Comparison of non-dimensional natural frequency obtained with Winkler and Pasternak foundation models corresponding to different values of aspect ratio

\section{Conclusion}

In the present study, free vibrational response of embedded SLGSs is investigated. To this end, Eringen's nonlocal elasticity continuum is incorporated into the various plate theories namely as classical plate theory (CLPT), first order shear deformation theory (FSDT), and higher order shear deformation theory (HSDT) to consider the sizeeffects on the vibration analysis of SLGSs. Both Winkler and Pasternak elastic foundation models are employed to represent the surrounding elastic medium. Explicit expressions are derived through an analytical solution to evaluate the natural frequencies corresponding to each type of nonlocal plate model.

Selected numerical results are presented to indicate the influence of the values of nonlocal parameter, Winkler modulus parameter, Pasternak modulus parameter, mode number, aspect ratio, and the type of nonlocal plate theory, in detail. It is observed that in contrast to the implementation of nonlocality which causes to reduce the stiffness of SLGS, by taking into account of elastic foundation, the natural frequency increases for all values of nonlocal parameter and this effect is more significant for higher aspect ratios relevant to all mode numbers which is again in contrast to the nonlocality effect that is more considerable in the lower aspect ratios. Also, it is found that the fundamental frequency of embedded SLGSs simulated by Pasternak foundation model is relatively more than the ones simulated by Winkler foundation model and this difference is approximately similar for all types of nonlocal plate theories. Moreover, it is observed that the difference between the two types of elastic foundation model is more prominent for higher values of nonlocal parameter and aspect ratio.

\section{References}

1. Lian, P.; Zhu, X.; Liang, S.; Li, Z.; Yang, W.; Wang, H. 2010. Large Reversible Capacity of High Quality Graphene Sheets as an Anode Material for Lithium-Ion
Batteries, Electro chimica Acta 55(12): 3909-3914, http://dx.doi.org/10.1016/j.electacta.2010.02.025.

2. Wu, Z.P.; Wang, J.N. 2009. Preparation of Large-Area Double-Walled Carbon Nanotube Films and Application as Film Heater, Physica E 42(1): 77-81, http://dx.doi.org/10.1016/j.physe.2009.09.003.

3. Kitipornchai, S.; He, X. Q.; Liew, K.M. 2005. Continuum Model for the Vibration of Multilayered Graphene Sheets, Physical Review B 72(7): 075443. http://dx.doi.org/10.1103/PhysRevB.72.075443.

4. Liew, K.M.; He, X.Q.; Kitipornchai, S. 2006. Predicting Nanovibration of Multi-Layered Graphene Sheets Embedded in an Elastic Matrix, ActaMaterialia 54(16): 4229-4236. http://dx.doi.org/10.1016/j.actamat.2006.05.016.

5. Azizi, S.; Safaei, B.; Fattahi, A. M.; Tekere, M. 2015. Nonlinear Vibrational Analysis of Nanobeams Embedded in an Elastic Medium including Surface Stress Effects, Advanced in Materils Since and Engineering: 1-7. http://dx.doi.org/10.1155/2015/318539.

6. Azizi, S.; Fattahi, A. M.; Kahnamouei, J. T. 2015. Evaluating mechanical properties of nanoplatelet reinforced composites under mechanical and thermal loads, Computational and Theoretical Nanoscience 12: 41794185.

7. Shen, L.; Shen, H.S.; Zhang, C. L. 2010. Nonlocal Plate Model for Nonlinear Vibration of Single Layer Graphene Sheets in Thermal Environment, Computational Materials Science 48(3): 680-685. http://dx.doi.org/10.1016/j.commatsci.2010.03.006.

8. Pradhan, S.C.; Phadikar, J.K. 2009. Small Scale Effect on Vibration of Embedded Multilayered Graphene Sheets based on Nonlocal Continuum Models, Physics Letters A 373 (11): 1062-1069. http://dx.doi.org/10.1016/j.physleta.2009.01.030.

9. Narendar, S.; Gopalakrishnan, S. 2009. Nonlocal Scale Effects on Wave Propagation in Multi-Walled Carbon Nanotubes, Computational Materials Science 47(2): 526-538.

http://dx.doi.org/10.1016/j.commatsci.2009.09.021.

10. Murmu, T.; Pradhan, S.C. 2009. Thermo-Mechanical Vibration of a Single-Walled Carbon Nanotube Embedded in an Elastic Medium based on Nonlocal Elasticity Theory, Computational Materials Science 46(4): 854859.

http://dx.doi.org/10.1016/j.comatsci.2009.04.019.

11. Sahmani, S.; Fattahi, A.M. 2017. An anisotropic calibrated nonlocal plate model for biaxial instability analysis of 3D metallic carbon nanosheets using molecular dynamics simulations, 4 (6): .1-14, http://dx.doi.org/10.1088/2053-1591/aa6bc0.

12. Fattahi, A. M.; Sahmani, S. 2017. Nonlocal temperature-dependent postbuckling behavior of FG-CNT reinforced nanoshells under hydrostatic pressure combined with heat conduction, Microsystem Technologies, Microsystem Technologies 23 (10): 5121-5137. http://dx.doi.org/10.1007/s00542-017-3377-x.

13. Fattahi, A.M.; Najipour, A. Experimental study on mechanical properties of PE / CNT composites, Journal of Theoretical and Applied Mechanics 55(2): 719-726, http://dx.doi.org/10.15632/jtam-pl.55.2.719.

14. Sahmani, S.; Fattahi, A.M. 2016. Size-dependent nonlinear instability of shear deformable cylindrical nano- 
panels subjected to axial compression in thermal environments, Microsystem Technologies 23(10): 47174731.

http://dx.doi.org/10.1007/s00542-016-3220-9.

15. Fattahi, A.M.; Safaei, B. 2017. Buckling analysis of CNT-reinforced beams with arbitrary boundary conditions, Microsystem Technologies 23(10): 5079-5091. http://dx.doi.org/10.1007/s00542-017-3345-5.

16. Sahmani, S.; Fattahi, A.M. 2017. Calibration of developed nonlocal anisotropic shear deformable plate model for uniaxial instability of 3D metallic carbon nanosheets using MD simulations, Computer Methods in Applied Mechanics and Engineering, 322: 187-207. http://dx.doi.org/10.1016/j.cma.2017.04.015.

17. Sahmani, S.; Fattahi, A.M. 2017. Development an efficient calibrated nonlocal plate model for nonlinear axial instability of zirconia nanosheets using molecular dynamics simulation, J. Mol. Graph. Model 75: 20-31. http://dx.doi.org/10.1016/j.jmgm.2017.04.018.

18. Kaveh E. Torkanpouri; Hassan Zohoor; Moharam H. Korayem. 2017. Global sensitivity analysis of backside coating parameters on dynamic response of AMAFM, MECHANIKA 22(3):282-290.

http://dx.doi.org/10.5755/j01.mech.23.2.13908.

19. Ali Yousefzadi Nobakht; Seungha Shin; Kenneth D Kihm; Drew C Marable; Woomin Lee. 2017. Heat flow diversion in supported graphene nanomesh, Carbon 123: 45-53. http://dx.doi.org/10.1016/j.carbon.2017.07.025.

20. Rasool Moradi-Dastjerdi; Hamed Momeni-Khabisi. 2017. Vibrational behavior of sandwich plates with functionally graded wavy carbon nanotube-reinforced face sheets resting on Pasternak elastic foundation, Journal of Vibration and Control. http://dx.doi.org/10.1177/1077546316686227.

21. Winkler, E. 1867. in: Theory of Elasticity and Strength, Dominicus, Prague.

22. Pasternak, P.L. 1954. On a New Method of Analysis of an Elastic Foundation by means of Two Foundation Constants, Gos, Izd, Lip, po Strait I Arkh.

23. Pradhan, S.C.; Murmu, T. 2010. Small Scale Effect on the Buckling Analysis of Single-Layered Graphene Sheet Embedded in an Elastic Medium based on Nonlocal Plate Theory, Physica E 42(5): 1293-1301. http://dx.doi.org/10.1016/j.physe.2009.10.053.

24. Liew, K.M.; He, X.Q.; Kitipornchai, S. 2006. Predicting Nanovibration of Multi-Layered Graphene Sheets Embedded in an Elastic Matrix, Acta Materialia 54(16): 4229-4236. http://dx.doi.org/10.1016/j.actamat.2006.05.016.
25. Eringen, A.C. 1972. Linear Theory of Nonlocal Elasticity and Dispersion of Plane Waves, International Journal of Engineering Science 10(5): 425-435. http://dx.doi.org/10.1016/0020-7225(72)90050-X.

26. Eringen, A.C. 1983. On Differential Equations of Nonlocal Elasticity and Solutions of Screw Dislocation and Surface Waves, Journal of Applied Physics 54(9): 47034710. http://dx.doi.org/10.1063/1.332803.

27. Reddy, J.N. 2007. Nonlocal theories for bending, buckling and vibration of beams, International Journal of Engineering Science V45:288-307. http://dx.doi.org/10.1016/j.ijengsci.2007.04.004.

Babak Safaei, A.M. Fattahi

FREE VIBRATIONAL RESPONSE OF SINGLELAYERED GRAPHENE SHEETS EM-BEDDED IN AN ELASTIC MATRIX USING DIFFERENT NONLOCAL PLATE MODELS

S u m m a r y

In this paper, the small scale effects are incorporated into the free vibration analysis of single-layered graphene sheets (SLGSs) embedded in an elastic medium. To this end, Eringen's nonlocal elasticity continuum are applied to the different types of plate theory namely as the classical plate theory (CLPT), first order shear deformation theory (FSDT), and higher order shear deformation theory (HSDT). Winkler and Pasternak foundation models used to simulate the surrounding elastic medium are compared with each other. Explicit expressions are derived to calculate the natural frequencies of square SLGSs corresponding to each type of nonlocal plate model. Selected numerical results are given to indicate the influence of the nonlocal parameter, Winkler and Pasternak elastic moduli, mode number, and the side length of SLGSs in detail. Also, comparison is made between the vibrational responses of SLGSs obtained through different nonlocal plate theories. It is found that the elastic foundation and value of nonlocal parameter have quite significant effects on the natural frequencies of SLGSs and these effects are influenced by mode number as well as side length.

Keywords: graphene sheets; free vibrations; nonlocal elasticity; exact solution; elastic foundation.

Received April 30, 2016 Accepted October 13, 2017 